\title{
AM: Uma Plataforma Digital para Criacao e Uso de Veículos de Comunicacao Locativos para Atividades Pedagógicas
}

\author{
Bernard C. Pereira ${ }^{1}$, Orivaldo de L. Tavares ${ }^{1}$, Renan F. Almeida ${ }^{1}$, Credine S. Menezes ${ }^{2}$ \\ ${ }^{1}$ Departamento de Informática - Universidade Federal do Espírito Santo (UFES) \\ Vitória - ES - Brasil \\ ${ }^{2}$ Instituto de Informática - Universidade Federal do Rio Grande do Sul \\ Porto Alegre - RS - Brasil \\ bernard.pereira@dataprev.gov.br, tavareseinf.ufes.br, ralmeida@ifes.edu.br \\ credine@gmail.com
}

\begin{abstract}
This article presents the platform "Learning in Motion"(AM) that enables the creation and use of communication vehicles based on location (VCLoc), with flexible rules of content manipulation, for use in educational activities in spaces where there may be movement, possibly outside the classroom. The following components of the platform AM are presented: 1) the "Communication Vehicles"Editor that allows the configuration of digital resources to the pedagogical architecture created by the teacher; 2) Mobile Client that allows students to navigate the VCLoc and if it was planned, interact with virtual objects in certain areas; 3) Educational Panel that allows the tracking of learners by the teacher.
\end{abstract}

Keywords: learning in motion; communication vehicle based on location; mobile application.

Resumo. Este artigo apresenta a plataforma "Aprendizagem em Movimento"(AM) que permite a criação e uso de veículos de comunicação baseados em localização (VCLoc), com regras flexíveis de manipulação de conteúdo, para o uso em atividades pedagógica em espaços em que possa haver movimento, possivelmente fora da sala de aula. Os seguintes componentes da plataforma AM são apresentados: 1) o Editor de "Veículos de Comunicação"que permite a configuração de recursos digitais necessários para à Arquitetura Pedagógica criada pelo professor; 2) o Cliente Móvel que permite aos alunos navegarem no VCLoc e, se tiver sido planejado, interagir com objetos virtuais em certos espaços; 3) o Painel Pedagógico que permite o acompanhamento dos aprendizes pelo professor.

Palavras-chaves: aprendizagem em movimento; veículo de comunicação baseado em localização; aplicativo móvel.

\section{Introdução}

As atividades desenvolvidas em espaços abertos, apresentam diversas oportunidades de aprendizado, podendo auxiliar os participantes na compreensão da relação que existe entre os conceitos teóricos, adquiridos em sala de aula, com os conhecimentos práticos adquiridos pela experiência prática, conhecido como conhecimento empírico. 
Considerada uma estratégia de ensino, tirar os alunos da escola para passeios, excursões ou visitas à exposições, cria oportunidades de descobertas de novidades, ampliando o interesse e fortalecendo a aprendizagem. O professor assume o papel de facilitador da aprendizagem, ao propor para a turma atividades pedagógicas motivadoras e inéditas para os aprendizes.

Aulas de campo, para não serem apenas um empirismo, devem articular-se à forma-ção teórica e a relação entre conhecimentos práticos e teóricos. Elas tem como objetivo ampliar o aproveitamento dos conhecimentos adquiridos, associando as abstrações men-tais aos aspectos físicos e naturais dos lugares explorados nas atividades desenvolvidas em espaços abertos. [Seniciato and Cavassan 2004] mostram, em um estudo feito com 97 alunos da sexta série do ensino médio, que a realização de aula de campo sobre botânica, após as aulas teóricas, trouxe ganhos de aprendizagem nos conceitos estudados, chegando a um aumento de $40 \%$ no rendimento dos conhecimentos adquiridos pelos alunos.

Experiência similar foi descrita em [Oliveira and Correia 2013], onde alunos do $3 o$ ano do ensino médio participaram de uma atividade cujo o objetivo era investigar o papel da aula de campo como mecanismo facilitador no processo de ensino-aprendizagem sobre o ecossistema recifal, importante ambiente do litoral de Alagoas, segundo [Oliveira and Correia 2013]:

"A partir dos resultados obtidos nos questionários aplicados nas duas fases, observou-se que os estudantes tiveram uma mudança considerável na aquisição de conhecimentos quando comparados os dados antes e após a aula de campo."

Além das oportunidades de aprendizagem, abordagens pedagógicas que se utilizam de aulas de campo também trazem desafios tais como: registro dos conhecimentos adquiridos, gerenciamento das atividades conduzidas, delimitação do espaço a ser explorado, monitoramento do percurso feito pelos participantes, entre outros.

Para auxiliar o professor e os alunos nas atividades de campo e nos desafios dessa abordagem pedagógica, a plataforma AM emprega recursos digitais providos por sistemas web e disponíveis em dispositivos móveis dotados de: sensores de GPS, sensores fotográficos, sensor de captação de áudio e internet. Esses dispositivos potencializam as oportunidades de aprendizado em atividades desenvolvidas em espaços abertos.

Os objetivos do projeto Plataforma AM são:

(i) estudar a utilização de Veículos de Comunicação Locativos (VCLoc) em Arquiteturas Pedagógicas (AP); (ii) especificar, projetar e implementar a plataforma AM para criar, via configura-ção, manipular e monitorar veículos de comunicação locativos; (iii) validar a plataforma AM com a criação de aplicações de VCLocs para suporte à atividades de aprendizagem em campo.

Este trabalho apresenta a Plataforma Aprendizagem em Movimento (seção 2 ), com uma visão geral do protótipo implementado. A seção 3 discute brevemente os conceitos que norteiam o projeto. Posteriormente, os 3 subsistemas que fazem parte da plataforma AM são apresentados: o Editor de Veículos de Comunicação (seção 4); o Cliente 
Móvel (seção 5) e o Painel Pedagógico (seção 6). O artigo finaliza na seção 7 com algumas considerações finais sobre este trabalho.

\section{Plataforma Aprendizagem em Movimento}

A plataforma Aprendizagem em Movimento (AM), cuja arquitetura é apresentada na Figura 1, é um projeto que tem como objetivo fornecer recursos digitais para atividades pedagógicas em aulas de campo, tais como passeios em espaços abertos. Composta por 3 subsistemas, um educador pode criar um Veículo de Comunicação baseado em Localização (VCLoc), usando o Editor de VCLoc (EdVCloc). Esse editor é apresentado na seção 4. Ele permite também a criação flexível de regras sobre a localização dos usuários e sobre os conteúdos publicáveis.

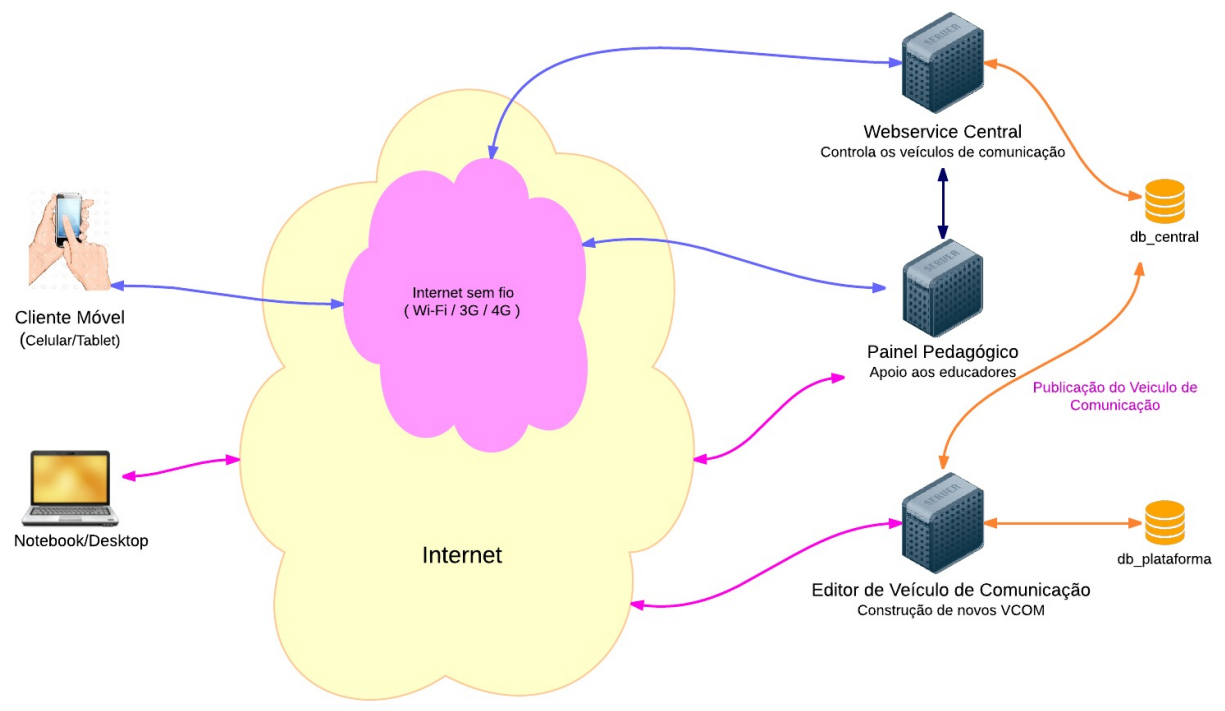

Figura 1. Visão Geral da Plataforma Aprendizagem em Movimento

Criado um VCloc no ambiente AM, os alunos podem acessar o Cliente Móvel, utilizando um aplicativo para celular ou tablet, que permite a interação com os objetos virtuais, adicionados ao ambiente pelos próprios estudantes a medida que navegam no mapa, conforme as regras previamente estabelecidas em cada Área de Interação definida pelo professor.

O painel pedagógico é um sistema web, com interface responsiva, para ser acessível utilizando dispositivos móveis. Nele o professor pode monitorar os eventos ocorridos no VCLoc, acompanhando os alunos durante a aula (seção 6).

\section{Uso de Veículos de Comunicação Baseados em Localização nas Arquiteturas Pedagógicas}

O paradigma pedagógico chamado de Arquitetura Pedagógica (AP) apoia-se na pedagogia da incerteza, pressupondo que o conhecimento nasce do movimento da dúvida, 
da incerteza, da busca de novas alternativas, do debate e da troca [Carvalho et al. 2005]. Buscando se diferenciar do ensino convencional de sala de aula e da materialização de apostilas de cursos para a educação à distância, uma AP abrange processos para a criação de novidades, descobertas e experimentações, centrada no ponto de vista do aprendiz, o protagonista da aprendizagem, durante a autoria de soluções individuais ou coletivas [Menezes et al. 2008].

As AP são estruturas de aprendizagem, que combinam abordagem pedagógica, inteligência artificial, educação a distância e concepção de tempo e espaço, e que são inspiradas em princípios das ideias construtivistas de Piaget e da pedagogia da pergunta de Freire, estabelecendo a Pedagogia da incerteza que implica em: (i) soluções de problemas reais, (ii) transformação de informações em conhecimento, (iii) autoria, expressão e interlocução, (iv) investigação e criação de novidades e (v) autonomia e cooperação [Carvalho et al. 2005].

Aplicativos de troca de mensagem, blogs da internet, redes sociais digitais, revista, televisão e rádio são alguns exemplos de Veículo de Comunicação (VCom). Um VCom é um canal para troca de comunicações entre pessoas que nele assumem papéis, as vezes simultaneamente, de criadores e consumidores de conteúdo [Pereira 2015].

Neste artigo, os Veículos de Comunicação (VComs) são digitais e são criados e acessados por meio da internet, tal como proposto no projeto MOrFEU (Multi Organizador Flexível de Espaços Virtuais) [Menezes et al. 2008], que possui características que permitem classificá-lo como um Gerenciador de Conteúdo (Content Management System - CMS). O projeto MOrFEU tem por objetivo a definição de conceitos e ferramentas que permitam a criação flexível de espaços virtuais, constituídos por VComs. Os VComs criados com as ferramentas desenvolvidas no âmbito do MOrFEU, são executados no mundo web, ou seja, em browser de internet, similarmente ao Joomla e ao WordPress.

A fotografia é um exemplo de UPI que mantem relação com o autor e é uma forma de expressão. Uma foto pode ser enviada para uma rede social, para um blog ou para uma sala de chat, por exemplo, cada um desses destinos pode servir como analogia para diferentes VComs, cujos conteúdos públicados são as diferentes UPIs, como a fotografia e outros textos escritos ou em outras mídias (áudio, vídeo e etc). Os VComs podem disciplinar os critérios para a recepção e publicação de UPIs.

A grande relevância no uso de Veículos de Comunicação (VCom) em Arquiteturas Pedagógicas (AP) está na questão da autoria, da expressão e da interlocução. [Menezes et al. 2008] propõem o conceito de UPI (Unidade de Produção Intelectual) como elemento básico de autoria para o registro de produções do usuário, e que pode, a qualquer momento, ser publicada em um Vcom. O projeto MorFEU propôs um ambiente para a construção de VCom, de modo flexível, pautada na plasticidade, ergonomia e na redução da repetição e da sobrecarga cognitiva. O presente trabalho dá continuidade a essas ideias e propõe os Veículos de Comunicação Locativos (VCLocs).

Um LBS (Location Based System) é uma categoria de serviço/sistema onde a posição corrente do usuário, ou do dispositivo, é usada no processamento, com impacto direto nos resultados obtidos, alterando as entradas e saídas de informações, podendo considerar a participação ativa ou não do usuário [Schiller and Voisard 2004]. 
[Pereira et al. 2014] definem o conceito de "Veículo de Comunicação Baseado em Localização" (VCLoc) como um VCom que funciona como um LBS, de modo que as interações usuário-sistema e usuário-usuário são afetadas pelo posicionamento geográfico do usuário.

Dar importância à localização do usuário para contextualizar o conteúdo das interações usuário-aplicação, permite o desenvolvimento de aplicações em que, por exemplo, quando o usuário estiver em um Jardim Zoológico, próximo das jaulas dos felinos, ele possa receber informações sobre os grandes felinos; quando ele estiver próximo das áreas destinadas às serpentes, ele possa receber informações sobre as serpentes existentes no zoológico e assim por diante.

Usar VCLoc em práticas pedagógicas permite ao educador motivar os aprendizes com experiências inovadoras de aprendizado. A plataforma AM possibilita a realização de diversas arquiteturas pedagógicas (APs) que prevejam interações entre usuários com o uso do VCLoc. Um VCLoc se destaca pela capacidade de permitir a criação de mundo virtual geograficamente delimitado, com regras de publicações e respostas adequadas ao planejamento de alguma AP.

A Tabela 1 é apresenta uma comparação da plataforma AM com outros sistemas que também tenham o objetivo de ajudar o educador a construir Veículos de Comunicação.

Na coluna "Projetado para" é apresentado o(s) modo(s) de uso do sistema, "Móvel" significa que o sistema foi desenvolvido para usar recursos de dispositivos móveis, ja "Web" define que o sistema foi construído para usar recursos da Web. A coluna "Principal Uso" apresenta o propósito principal de construção do sistema. A coluna "Locatividade" define se o sistema utiliza informação de posição geográfica para acessar o sistema ou para processar e disponibilizar informação associada a localização do usuário e seu registro histórico de deslocamento.

\begin{tabular}{llll} 
& \multicolumn{2}{l}{ Tabela 1. Comparativo entre os sistemas. } & \\
Sistema & Projetado para & Principal uso & Locatividade \\
Joomla & Web & Sites em Geral & Não \\
MOrFEu & web & Aplicações Educacionais & Não \\
Aris Games & Móvel & Jogos & Sim \\
Plataforma AM & Móvel & Aplicações Educacionais & Sim
\end{tabular}

A plataforma ARIS, disponível em http: / / arisgames.org assim como a plataforma AM também é projetada para dispositivos móveis e tem suporte a locatividade. Entretanto, a plataforma ARIS segue uma orinteção conceitual diferente que são os Jogos Educacionais, já a plataforma AM usa o conceito e o paradigma dos Veículos de Comunicação.

\section{Editor de Veículos de Comunicação Baseados em Localização}

Para usar um VCLoc em uma AP, é necessário que o professor possua meios de criar um VCLoc, sem o uso de programação. A Figura 2 apresenta um VCLoc, composto de 3 áreas de interação, e que foi construído com o uso do Editor de VCLoc, uma ferramenta web, disponível em editor.plataformaam.com. 


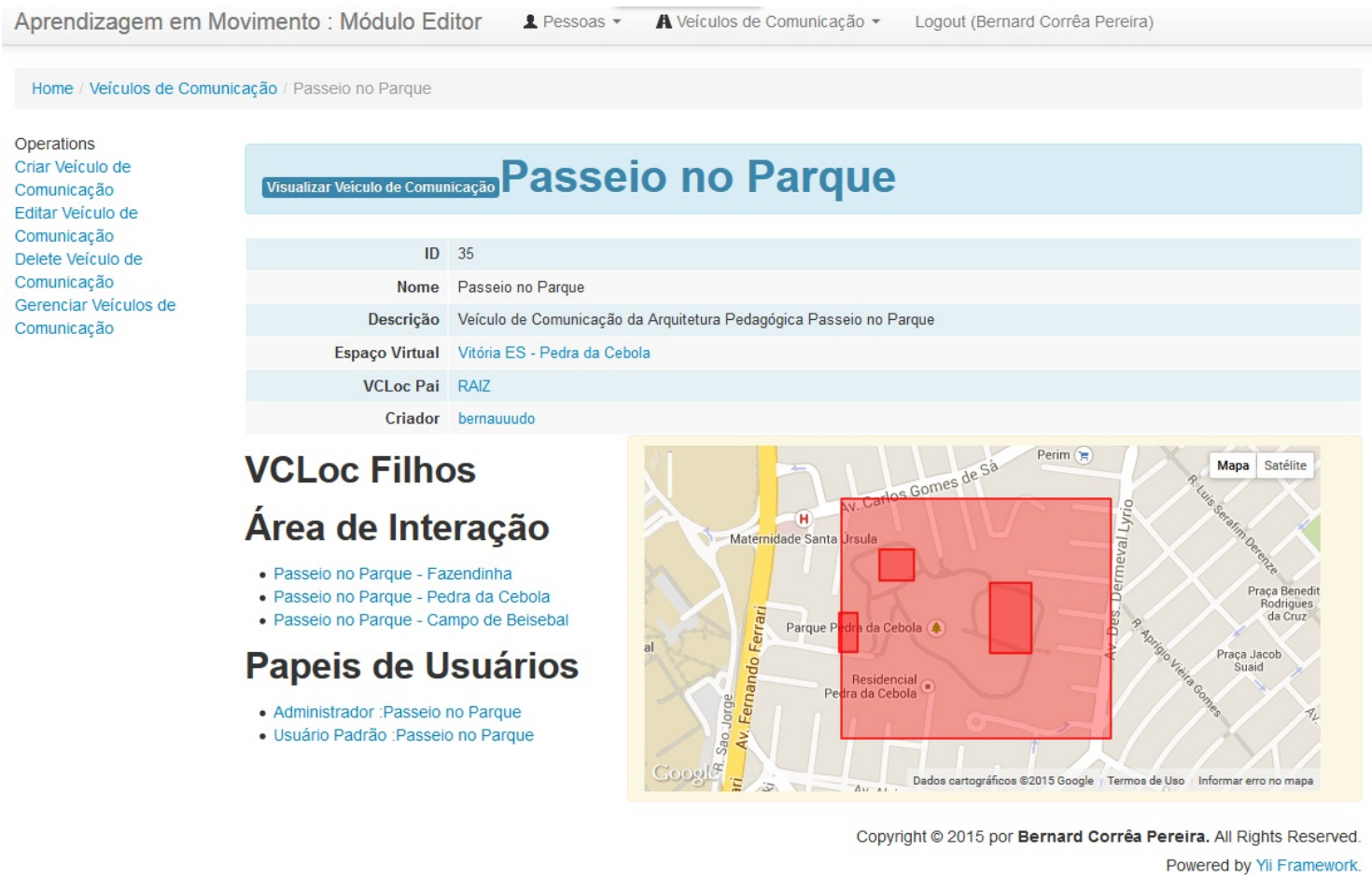

Figura 2. Editor de Veículo de Comunicação

O retângulo maior na Figura 2 representa a área geográfica de atuação do VCLoc. Os retângulos menores são Áreas de Interação (AI), onde os professores associam regras de publicação e de respostas, as quais determinam:

(i) Qual tipo da UPI pode ser utilizado na interação;

(ii) Se a interação com o ambiente virtual requer a presença física do usuário, e qual é a distância em metros necessária para que o elemento virtual se torne visível ao usuário.

(iii) Quais papéis de usuários podem usar essa regra.

Para cada AI o professor pode criar um conjunto de regras para publicações diferentes, como por exemplo, "regra de publicação 01: usuários somente podem publicar fotos quando estiverem presentes na área de interação”. Para cada regra de publicação, várias regras de respostas podem ser associadas. Por exemplo: "As respostas à publicação da regra de publicação 01 podem ser feitas com texto escrito, por usuários fisicamente presentes na área de interação" e "As respostas à publicação da regra de publicação 01 podem ser feitas com fotos, por usuários que não precisam estar fisicamente presentes na área de interação".

Ao criar um VCLoc, o editor gera automaticamente os papéis Administrador (com poderes de edição do VCLoc) e Usuário Padrão (para ser selecionado no dispositivo móvel). O professor pode criar diversos papéis de usuários, associando-os a conjuntos de regras de publicação e de resposta diferentes. A flexibilidade de criação dos VCLoc permite a realização de um grande número de atividades pedagógicas em espaços abertos, utilizando dispositivos móveis, celulares e tablets. 


\section{Cliente Móvel para Veículos de Comunicação Baseados em Localização}

Ao se inscrever em um VCLoc, o usuário escolhe um dos papéis disponíveis para a seleção. O papel escolhido determina como o estudante interage com o VCLoc. A Figura 3 mostra a navegação em um veículo de comunicação. O estudante é representado por um avatar e as publicações, visíveis ao usuário, são representadas por caixas de diálogos.

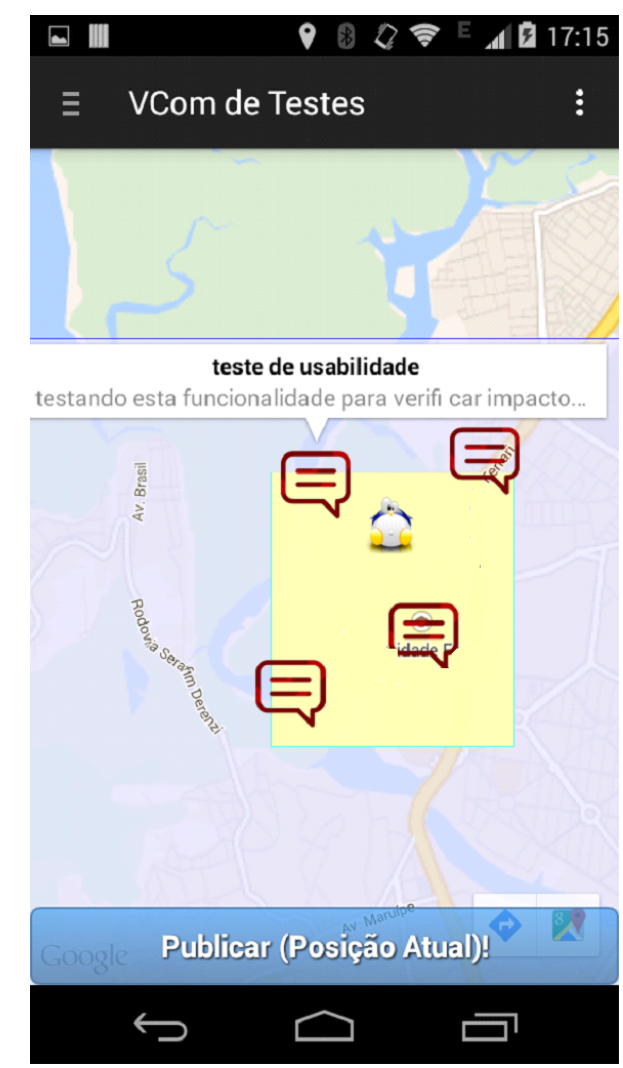

Figura 3. Cliente Móvel para Manipulação de Veículo de Comunicação

O usuário pode criar, acessar, comentar e responder publicações (UPIs), dentro de uma área de interação do VCLoc. Podendo visualizar as regras de publicações de uma determinada área de interação.

Atualmente o aplicativo celular se encontra disponível para dispositivos que executam sistema operacional Android.

\section{O Monitoramento de Veículos de Comunicação Baseados em Localização com o Painel Pedagógico}

Os diversos alunos que um professor possui podem se distanciarem do grupo, sumir da visão dos monitores, ou se perderem. A Figura 4 mostra a tela de acompanhamento online dos estudantes feita por meio de um mapa, no qual o professor tem a capacidade de visualizar os alunos que se encontram dentro do VCLoc.

O painel pedagógico também traz informações sobre as interações ocorridas em um VCLoc, exibindo no mapa as publicações e suas respostas, filtradas por alunos [Pereira 2015]. 


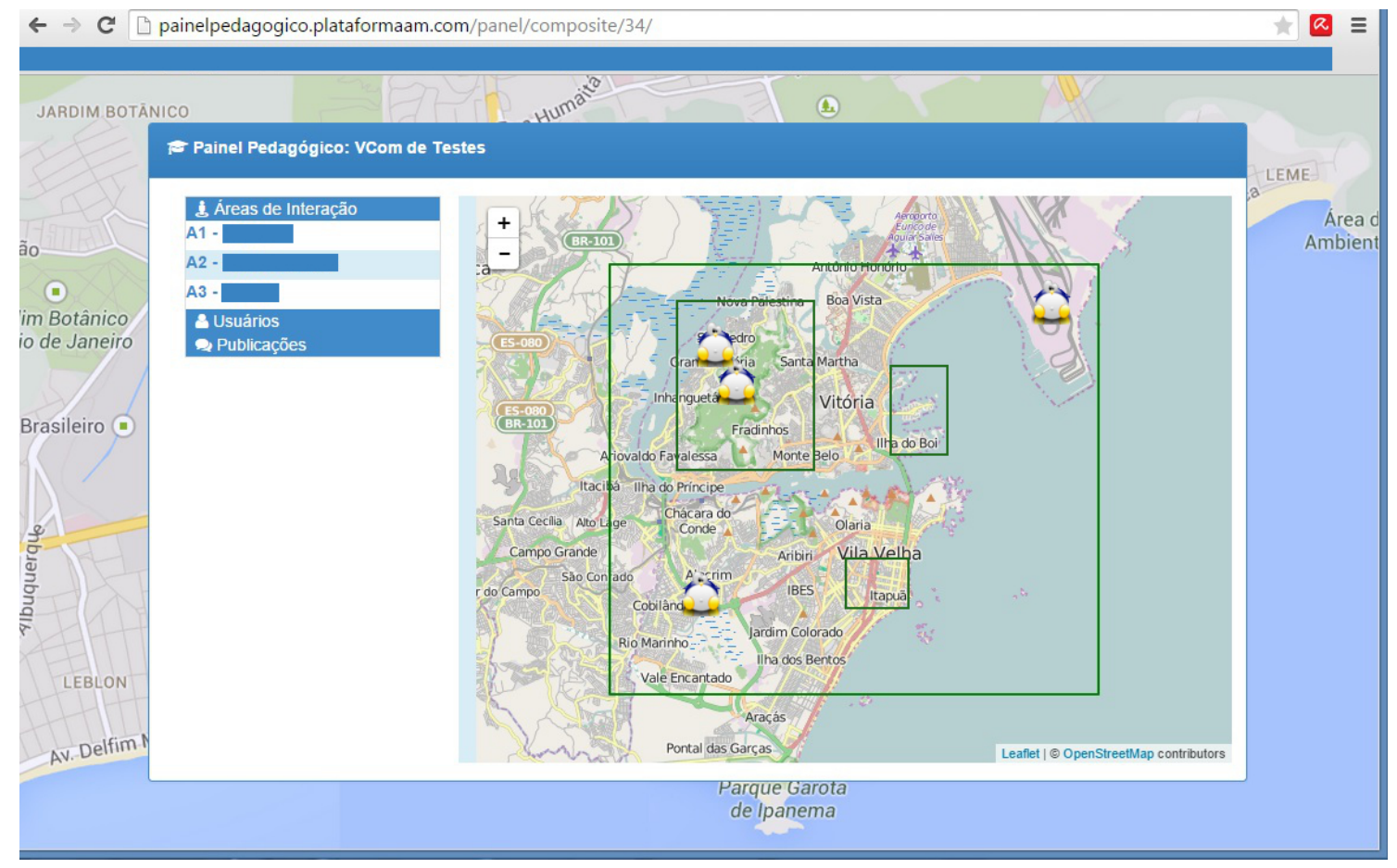

Figura 4. Painel Pedagógico: Monitoramento do Veículo de Comunicação

\section{Considerações finais}

Este artigo apresentou uma plataforma digital para a criação e uso de aplicações web que contextualizam suas interações com os usuários considerando a localização dele. Essas aplicações são chamadas de Veículos de Comunicação Locativos (VCLocs).

O código fonte da plataforma AM está disponível, sob licença LGPL, em: https://github.com/LocationBasedCMS/. A implementação atual permite a manipulação de texto escritos e fotos. A adição de outras mídias processáveis depende apenas da implementação de mecanismo de processamento, o que enriquecerá as experiências de uso do ambiente: vídeos, áudio, fotos esféricas, entre outras.

Em [Pereira 2015] existe um plano para a realização de experiências de modo a compreender melhor o uso de VCLoc. Esse plano inclui a realização de testes, dividindo em grupos de 3 turmas similares de estudantes, ou seja, do mesmo ano e mesmo perfil social:

(i) a primeira turma utiliza o Cliente Móvel (CliVCLoc) para uma aula de campo; móveis;

(ii) a segunda turma procedendo com uma aula de campo sem apoio de aplicativos

(iii) a terceira turma procedendo com uma aula tradicional em sala de aula.

Após as aulas planejadas pelas 3 APs, um questionário elaborado por equipe multidisciplinar, composta por professores, pedagogos e outros profissionais avaliariam o interesse despertado nos alunos, o aprendizado conseguido e pistas do efeito alcançado [Pereira 2015]. Esta é apenas uma das possibilidades de experimentos a serem realizados para compreensão do uso de VCLoc em APs. Apesar do otimismo que desperta, pode não 
ser capaz de superar barreiras como a experiência de uso do aplicativo móvel, o adequado planejamento de uma AP ou a imposição de resultados quantitativos (notas dos alunos) que nem sempre refletem o aprendizado real de um aluno.

A realização do experimento ainda exigiria as (i) autorizações da instituição de ensino, alunos e responsáveis; (ii) apoio de professores e pedagogos; (iii) celulares de baixo custo com o sistema operacional Android ou compatível [Pereira 2015].

Como implementações futuras temos a inclusão de novas mídias além de textos escritos e fotos, a possibilidade de construir UPIs com a combinação das mídias suportadas pela plataforma AM e o desenvolvimento de um subsistema para criar relatos individuais das atividades desenvolvidas, de modo a combinar as múltiplas mídias produzidas durante uma atividade. Tal implementação permitirá ampliar ainda mais as oportunidades de aprendizagem, podendo contemplar alunos e professores com diferentes estilos de aprendizagem, que segundo propõem [Ocepek et al. 2013] tendem a preferir determinadas mídias em detrimento a outras, de acordo com o perfil individual.

Os testes realizados com a plataforma AM apresentada, ficaram restritos a um pequeno grupo de voluntários, sendo o experimento com um grupo maior de alunos e professores objeto de trabalhos futuros.

\section{Referências}

Carvalho, M. J. S., Nevado, R. A. d., and Menezes, C. d. (2005). Arquiteturas pedagógicas para educação a distância: concepções e suporte telemático. Anais do XVI Simpósio Brasileiro de Informática na Educação, Juiz de Fora-MG. Brasil.

Menezes, C. S., de Nevado, R. A., de Castro Jr, A. N., and Santos, L. N. (2008). Morfeumulti-organizador flexível de espaços virtuais para apoiar a inovação pedagógica em ead. In Anais do Simpósio Brasileiro de Informática na Educação, volume 1, pages 451-460.

Ocepek, U., Bosnić, Z., Šerbec, I. N., and Rugelj, J. (2013). Exploring the relation between learning style models and preferred multimedia types. Computers \& Education, 69:343-355.

Oliveira, A. and Correia, M. D. (2013). Aula de campo como mecanismo facilitador do ensino-aprendizagem sobre os ecossistemas recifais em alagoas. Alexandria Revista de Educação em Ciência e Tecnologia, 6(2):163-190.

Pereira, B., Tavares, O., and de Menezes, C. (2014). Aprendizagem em movimento: Um editor para a criação de veículos de comunicações locativos. In CSBC 2014 - III desafIE ().

Pereira, B. C. (2015). Aprendizagem em movimento - uma plataforma para a criaçção e uso de veiculos de comunicação na realização de arquiteturas pedagogicas. Master's thesis, Programa de pós graduação em em Informática da UFES, Vitória-ES.

Schiller, J. and Voisard, A. (2004). Location-based services. Elsevier.

Seniciato, T. and Cavassan, O. (2004). Aulas de campo em ambientes naturais e aprendizagem em ciências-um estudo com alunos do ensino fundamental field classes in natural environment and science learning-a study with students from elementary school. Ciência \& Educação, 10(1):133-147. 